\title{
Audit of the value of bone marrow aspirates when a bone marrow trephine is used for lymphoma staging
}

\author{
Zanna J. Voysey • Elizabeth J. Soilleux
}

Received: 27 February 2011 /Accepted: 28 March 2011/Published online: 13 April 2011

(C) Springer-Verlag 2011

\begin{abstract}
Bone marrow involvement is assessed in lymphoma staging/restaging following treatment/relapse by a combination of an aspirate and trephine biopsy. Of 262 consecutive cases staged by aspirate and trephine, the aspirate yielded a diagnosis when a trephine did not in only $11.8 \%$. Trephine biopsies were positive in $34.7 \%$ cases and equivocal/insufficient in $14.9 \%$, while aspirates were positive in $11.1 \%$ cases and equivocal/insufficient in $21 \%$. We suggest that in the majority of cases, it may be acceptable either to store an aspirate for reporting only if the trephine is inconclusive or to take a trephine biopsy without an aspirate.
\end{abstract}

Keywords Lymphoma · Bone marrow · Staging · Trephine · Aspirate $\cdot$ Correlation

$\begin{array}{ll}\text { Abbreviations } \\ \text { HL } & \text { Hodgkin lymphoma } \\ \text { NHL } & \text { Non-Hodgkin lymphoma } \\ \text { CLL } & \text { Chronic lymphocytic leukaemia } \\ \text { HCL } & \text { Hairy cell leukaemia }\end{array}$

Z. J. Voysey

Oxford University Medical School,

Oxford, UK

E. J. Soilleux

Department of Cellular Pathology, John Radcliffe Hospital, Oxford, UK

\section{E. J. Soilleux $(\square)$}

Nuffield Department of Clinical Laboratory Sciences,

Oxford University,

Oxford OX3 9DU, UK

e-mail: Elizabeth.soilleux@ndcls.ox.ac.uk

\section{Introduction}

Assessment of bone marrow (BM) involvement in lymphoma staging/restaging following treatment/relapse by a combination of an aspirate (BMA) and trephine biopsy (BMT) may affect prognosis and treatment modality [6]. Frequently, BMAs are reported by haematologists and BMTs by histopathologists in the UK, the two being correlated at clinicopathological meetings [4].

BMTs have the advantage of allowing immunostaining or polymerase chain reaction to highlight lymphoma cells, while BMA morphological interpretation may be operatordependent, giving a lower sensitivity [5]. BMTs are less frequently insufficient in fibrotic lymphomas, where aspiration gives a "dry tap" (no cells). BMAs may be excessively haemodiluted or may clot, giving insufficient preparations [1].

Few previous studies have investigated the relative contributions of BMA and BMT to diagnosis, most concentrating on Hodgkin lymphoma (HL) and demonstrating that BMT is considerably more sensitive at detecting BM involvement by lymphoma than BMA $[1,2,6]$. The few studies performed in non-Hodgkin lymphomas (NHL) have given more equivocal results. One study correlated flow cytometry, BMT and BMA results in 39 B-cell NHLs (BNHLs), concluding that BMT and flow cytometry both detected marrow infiltration by B-NHL more effectively than BMA [5]. BMT and BMA results correlated in $61.5 \%$ cases. Where the BMT was negative, the BMA was equivocal in $5.1 \%$ cases and positive in $1 / 39(2.6 \%)$ case and where the BMT was equivocal, the BMA was negative in all 5/39 $(12.8 \%)$ cases [5]. Another study of 50 NHLs showed that BMT and BMA results correlated in $80 \%$ cases and that $69 \%$ of ( $82 \%$ of indolent and $40 \%$ of aggressive) cases with a positive BMT also had a positive BMA [3]. In 10\%, BMT did not detect lymphoid infiltration despite a positive 
aspirate. This study concluded that BMA was a useful adjunct to BMT, giving more rapid results using simpler techniques, occasionally detecting BM involvement in cases with a negative BMT and, together with BMT, allowing for the examination of larger amounts of BM [3].

Given the small number of relatively low power studies with largely equivocal results in NHL, we undertook a large retrospective audit study to investigate the relative contributions of BMT and BMA to assessment of lymphoma stage.

\section{Methods}

A retrospective audit study of HL and NHL patients undergoing staging or restaging by both aspirate and trephine biopsy in our centre between January 2008 and December 2009 was restricted to cases that (1) had a definitive biopsy-proven diagnosis at an extramedullary site, (2) had both BMA and BMT for staging, (3) required no techniques other than immunostaining on the BMT for diagnosis and (4) had a low probability of a leukaemic phase (i.e. excluding chronic lymphocytic leukaemia/small lymphocytic lymphoma, acute lymphoblastic leukaemia/ lymphoblastic lymphoma and hairy cell leukaemia). Corresponding BMA and BMT results were categorised as positive, negative, equivocal or insufficient. A chi-squared test was used to determine whether there were significant differences between all four outcomes in BMT and BMA diagnoses, refined using a $2 \times 2$ contingency test to determine whether the number of positive and negative cases differed significantly between BMTs and BMAs, with all $p$ values at the $95 \%$ confidence level (www.statpages.org).

\section{Results}

Two hundred and sixty-two suitable patients were identified (Fig. 1a). Figure $1 \mathrm{~b}$ demonstrates the differences in positive,

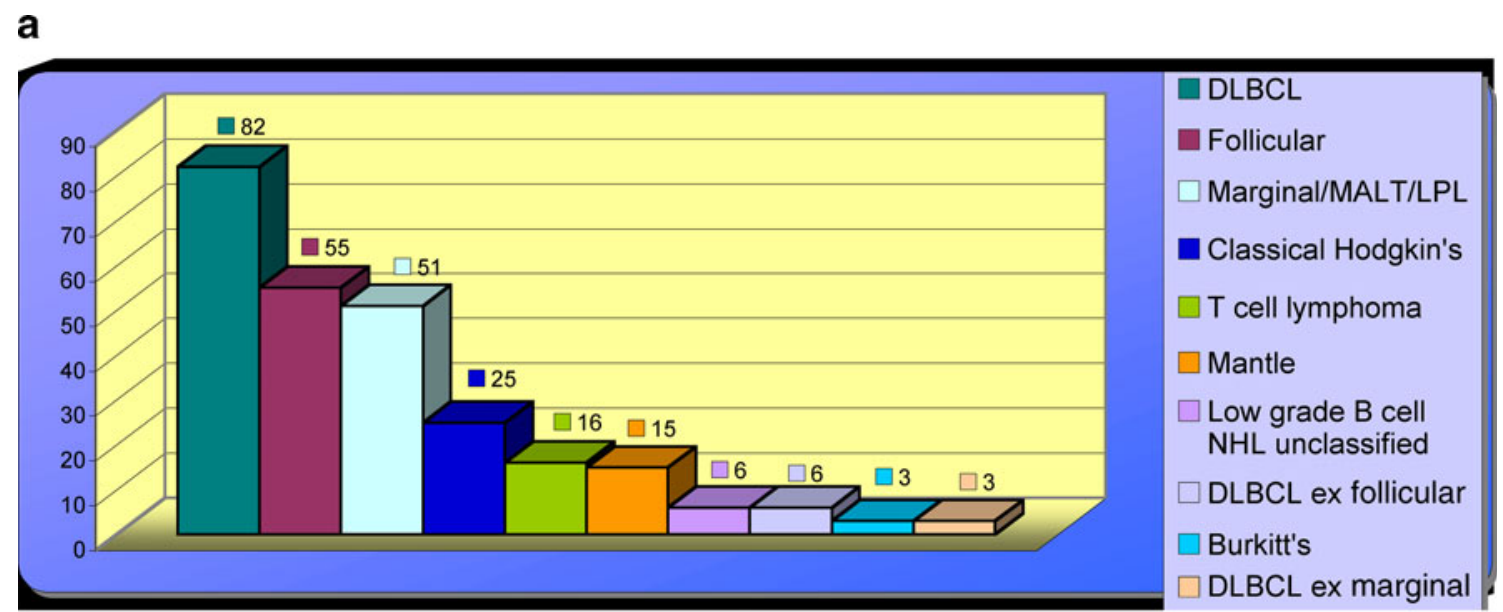

b

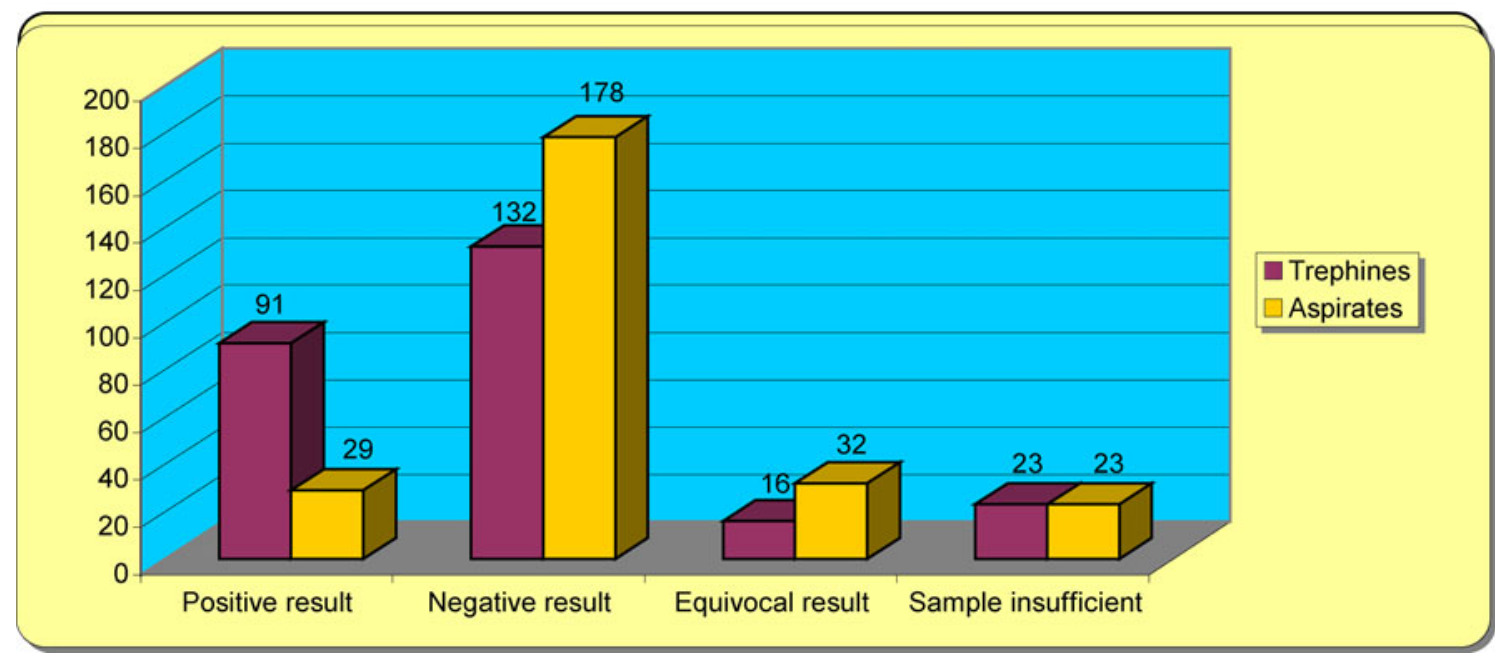

Fig. 1 a. Types of lymphoma included in the 262 cases in this study. b. Diagnostic categories for the 262 bone marrow trephine biopsies and aspirates included in this study 
a

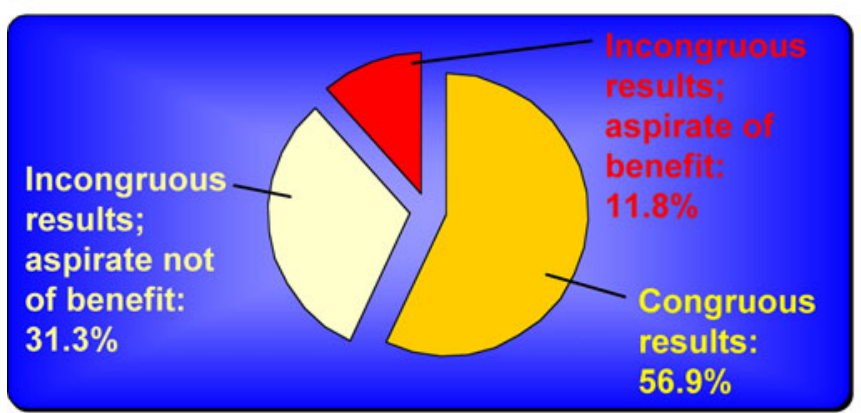

C

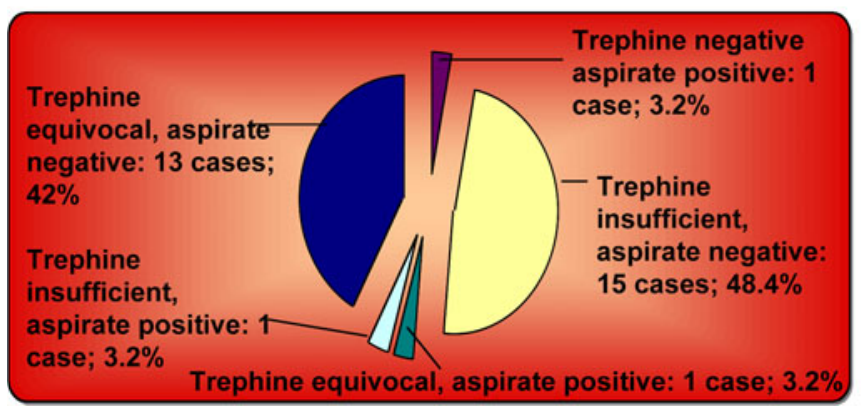

Fig. 2 a. Pie chart to show correlations and lack of correlation between aspirate and trephine biopsies, including the percentage of cases in which the aspirate conferred or did not confer additional diagnostic utility $(n=262)$. b. Diagnostic categories for trephines and

negative, equivocal and insufficient rates for BMTs and BMAs $(p<0.001)$. There was no difference in the number of insufficient samples $(23 / 262=8.8 \%)$ produced by the two techniques. BMAs produced twice as many equivocal results as BMTs $(32 / 262,12.2 \%$ versus $16 / 262,6.1 \%)$. BMTs yielded more positive $(91 / 262,34.7 \%$ versus $29 / 262,11.1 \%$; $p<0.001)$ and fewer negative $(132 / 262,50.4 \%$ versus $178 /$ $262,68.0 \% ; p<0.001)$ results than BMAs.

In 149/262 (56.9\%) cases, BMT and BMA results were concordant (Fig. 2a), 26/149 (17.4\%) being positive, 115/ 149 (77.2\%) being negative, 5/149 (3.4\%) being insufficient and $3 / 149(2.0 \%)$ being equivocal (Fig. 2b). Of 113/262 (43.1\%) cases in which BMT and BMA results failed to correlate, BMA provided a conclusive positive or negative result when BMT was either equivocal or insufficient in 31/ $262(11.8 \%)$ cases (Fig. 2c). A positive result was provided by BMA rather than BMT in only 3/262 (1.1\%) cases. In only $1(0.38 \%)$ case was BMT adequate but negative and BMA positive. BMA failed to correlate with BMT and did not contribute to assessment of BM involvement in 82/262 (31.3\%) cases (Fig. 2d). In 35/262 (13.4\%) of cases, BMA was negative while BMT was positive and in 29/262 (11\%) cases BMA was insufficient, equivocal or negative while BMT was positive. Broadly similar results are seen between different types of lymphoma in our study. For diffuse large B-cell lymphoma, samples were concordant in $48 / 82$ b

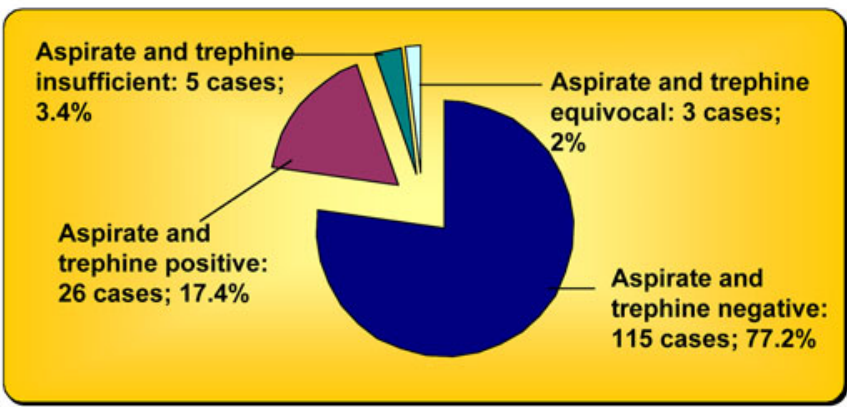

d

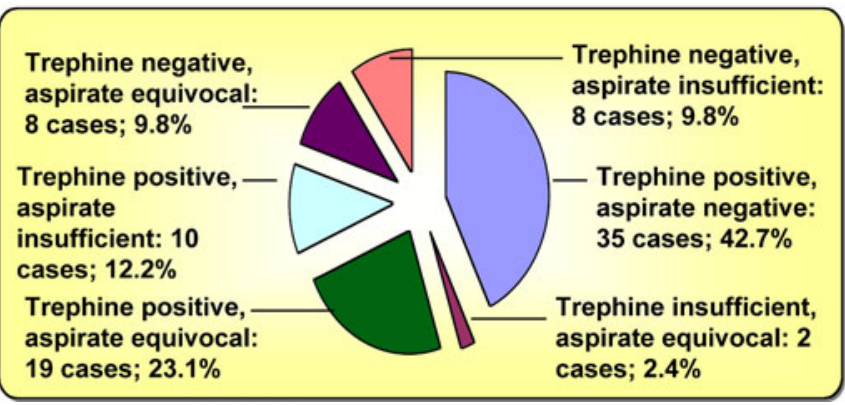

aspirates when results were congruent $(n=149)$. c and d. Diagnostic categories for trephines and aspirates when results were incongruent, but the aspirate conferred additional diagnostic utility $(\mathbf{c}, n=31)$ or did not confer additional diagnostic utility $(\mathbf{d}, n=82)$

(58.5\%) cases, discordant with aspirate being of benefit in $10 / 82(12.2 \%)$ and discordant with aspirate being of no benefit in $24 / 82$ (29.2\%). For follicular lymphoma, samples were concordant in $27 / 55(49.1 \%)$ cases, discordant with aspirate being of benefit in $7 / 55(12.7 \%)$ and discordant with aspirate being of no benefit in 21/55 (38.2\%). For marginal zone/lymphoplasmacytic lymphoma, samples were concordant in 23/51 (45.1\%) cases, discordant with aspirate being of benefit in 5/51 (9.8\%) and discordant with aspirate being of no benefit in 23/51 (45.1\%).

\section{Discussion}

Of the 113/262 (43.1\%) cases in which BMT and BMA did not correlate, BMA provided a conclusive positive/negative result when BMT was either equivocal/insufficient in 31/262 $(11.8 \%)$ cases. In only $1 / 262(0.38 \%)$ case was BMT adequate but negative, while BMA was positive, in keeping with a previous study [5]. Either a BMT could be performed without a BMA, or a BMA could be stored and only reported if the BMT is insufficient or equivocal. However, storing a BMA that might show evidence of lymphoma without reporting it, when BMT is reported as negative, raises complex medicolegal concerns. Because over half the BMTs in this study were reported as negative $(50.4 \%)$, it 
would be difficult to decide which of these cases merited BMA examination. Our data would support the suggestion of a previous study that, if BMA were not performed, sensitivity might be improved by bilateral BMTs with no BMA, rather than a unilateral BMT and BMA [5].

Conflicts of interest The authors state that they have no conflict of interest.

\section{References}

1. Howell SJ, Grey M, Chang J, Morgenstern GR, Cowan RA, Deakin DP, Radford JA (2002) The value of bone marrow examination in the staging of hodgkin's lymphoma: a review of 955 cases seen in a regional cancer centre. Br J Haematol 119(2):408-411. doi:3842[pii]
2. Moid F, De Palma L (2005) Comparison of relative value of bone marrow aspirates and bone marrow trephine biopsies in the diagnosis of solid tumor metastasis and Hodgkin lymphoma: institutional experience and literature review. Arch Pathol Lab Med 129(4):497-501. doi:OA4200[pii]

3. Musolino A, Guazzi A, Nizzoli R, Panebianco M, Mancini C, Ardizzoni A (2010) Accuracy and relative value of bone marrow aspiration in the detection of lymphoid infiltration in non-Hodgkin lymphoma. Tumori 96(1):24-27

4. NICE (2003) Guidance on cancer services - improving outcomes in haematological cancers - the manual

5. Sah SP, Matutes E, Wotherspoon AC, Morilla R, Catovsky D (2003) A comparison of flow cytometry, bone marrow biopsy, and bone marrow aspirates in the detection of lymphoid infiltration in $\mathrm{B}$ cell disorders. J Clin Pathol 56(2):129-132

6. Subramanian R, Basu D, Badhe B, Dutta TK (2007) Role of bone marrow trephine biopsy in the diagnosis of marrow involvement in Hodgkin's disease. Indian J Pathol Microbiol 50(3):640-643 